Pacific Journal of Mathematics

SURGERY OF INVOLUTIONS WITH MIDDLE-DIMENSIONAL

KarL HeInZ overman AND Reinhard Schultz 


\title{
SURGERY OF INVOLUTIONS WITH MIDDLE-DIMENSIONAL FIXED POINT SET
}

\section{Karl Heinz Dovermann and Reinhard Schultz}

\begin{abstract}
Surgery on manifolds with involution is considered when the dimension of the fixed point set is less than or equal to half the dimension of the ambient manifold. As in earlier work of the first author, there are some sharp contrasts with the corresponding results when strict inequality holds. The overall approach is based upon earlier work of the first author, but the results are reformulated in broader contexts reflecting both known and anticipated applications of the underlying ideas.
\end{abstract}

During the past quarter century surgery theory has proved to be a very powerful tool for studying group actions on manifolds (compare [5], [26]). Usually the strongest applications require a condition called the Gap Hypothesis; for actions of the group $\mathbf{Z}_{2}$, this condition says that the dimension of the fixed point set is strictly less than half the dimension of the ambient manifold with $\mathbf{Z}_{2}$-action. In [11] the first named author studied equivariant surgery problems in cases just beyond the realm of the Gap Hypothesis; specifically, in [11] the dimension of the fixed point set is exactly half the dimension of the ambient manifold. The ideas of [11] have subsequently found applications in several separate contexts (Schultz [28], Masuda [25], S.-i. Kakutani [19], Kitada [20], and DovermannMasuda-Schultz [12]). Many interesting applications are in cases that require modifications of the general theory. For example, it is often necessary to be flexible about the fundamental group and orientability properties of the fixed set. Although such extensions can often be verified in special cases by ad hoc discussions, for several reasons it is not always possible to justify the full generalizations by short, unambiguous references to [11]. Therefore it seems appropriate to establish explicit, uniform generalizations of the main results in [11] that will systematically cover the applications in the five papers mentioned as well as potential future applications. Some of the generalizations in this paper were proposed in the review of [11] by G. A. Anderson [1].

Comments and acknowledgments. We are grateful to S. Zdravkovska of Mathematical Reviews for providing a copy of the original typescript of the review of [11] by G. Anderson [1]. This made it possible to understand 
more completely some of the comments in the printed version. Several remarks in [1] and the original typescript were helpful in the preparation of this paper. In particular, we have attempted to address the comments in [1] regarding the wording of some results in [11].

Both S.-i. Kakutani and M. Masuda have been very generous in furnishing accounts of their work before publication. Comments by $\mathrm{S}$. Cappell and W. Weinberger have yielded improvements in the discussion of $\Gamma$-groups, and their advice in this connection has been very much appreciated.

1. Summary of results. Most of our conclusions will be stated for smooth involutions. Some remarks regarding analogs for other categories of $\mathbf{Z}_{2}$-manifolds are included immediately following the main part of this summary.

Roughly speaking, an equivariant surgery problem in our context is an equivariant degree 1 map $f: X \rightarrow Y$ between two smooth $\mathbf{Z}_{2}$-manifolds together with some additional technical data. Our main objective is to modify $f$ by a process called equivariant surgery in order to obtain a new equivariant map $f^{\prime}: X^{\prime} \rightarrow Y^{\prime}$ that is a homotopy equivalence (but not necessarily a $\mathbf{Z}_{2}$-homotopy equivalence). Such maps are called pseudoequivalences in [13].

To be more precise, our equivariant surgery problems will satisfy some additional restrictions. The reasons for the extra conditions will be discussed immediately following the definition.

Notation. Given a map $f: X \rightarrow Y$ between two smooth $\mathbf{Z}_{2}$-manifolds, let $F_{\alpha}$ denote the components of the fixed point sets of $Y$, and let $E_{\alpha}$ denote the union of all components of the fixed point set of $X$ that are mapped into $F_{\alpha}$ by $f$. The map induced by $f$ from $E_{\alpha}$ to $F_{\alpha}$ will be called $f_{\alpha}$. If $b: \xi \rightarrow \eta$ is a vector bundle isomorphism covering the identity on $X$ then the restriction $b_{\alpha}$ of $b$ to $E_{\alpha}$ splits into a direct sum $b_{\alpha}(+) \oplus b_{\alpha}(-)$, corresponding to the eigenspaces of \pm 1 (note that $\mathbf{Z}_{2}$ acts on each fiber as a linear involution and thus induces a fiberwise decomposition into eigenspaces). Finally, we define $N E_{\alpha}$ to be the normal bundle of $E_{\alpha}$ in $X$.

Definition. Let $f: X \rightarrow Y$ be a $\mathbf{Z}_{2}$-equivariant map of degree 1 between smooth, compact, 1-connected $\mathbf{Z}_{2}$-manifolds. Let $\xi$ be a $\mathbf{Z}_{2}$-vector bundle over $Y$ and $b: T X \rightarrow f^{*} \xi$ a stable $\mathbf{Z}_{2}$-vector bundle isomorphism. Finally, for each component $F_{\alpha}$ of the fixed point set of $Y$ let $\eta_{\alpha}$ be a vector bundle over $F_{\alpha}$, let $\dot{c}_{\alpha}: N E_{\alpha} \rightarrow f_{\alpha}^{*} \eta_{\alpha}$ be a vector bundle isomorphism, and let $\ddot{c}_{\alpha}$ be a stable equivalence between $\dot{c}_{\alpha}$ and $b_{\alpha}(-)$. 
Set $c$ equal to $(\dot{c}, \ddot{c})$. The data $(f, b, c)$ will be called a $\mathbf{Z}_{2}$-normal map (or equivalently a $\mathbf{Z}_{2}$-surgery problem) if the following conditions are satisfied:

(1.1a) Each of the sets $E_{\alpha}$ is nonempty; in other words, the induced map of fixed point set components is surjective.

(1.1b) If $F_{\alpha}$ is a component of the fixed point set of $Y$ and $E_{\beta}$ is a component of the fixed point set of $X$ that is mapped into $F_{\alpha}$, then the dimensions of $F_{\alpha}$ and $E_{\beta}$ are equal and the first Stiefel-Whitney classes of these components correspond under $f$.

(1.1c) The dimension of $Y$ is at least 5, and for each $\alpha$ either the dimension of $F_{\alpha}$ is at least 5 or else the map $f_{\alpha}$ is a $\mathbf{Z}_{2}$-homology equivalence.

(1.1d) The induced map of boundaries is a pseudoequivalence.

Many of these conditions are standard in surgery theory. For example, the dimension restrictions are the ones needed to carry through the usual surgery-theoretic techniques, and the low-dimensional assumptions are designed to ensure that everything is already satisfactory on such components. The conditions involving fixed point sets are necessary for the existence of a pseudoequivalence. Normal maps always involve some additional bundle data, and the $\mathbf{Z}_{2}$-vector bundle map $b$ corresponds exactly to what one has in the nonequivariant case.

The role of the unstable data $c$ is more subtle. In nonequivariant surgery theory there is an important characterization of normal maps in terms of homotopy-theoretic invariants (compare [4] or [30]). This follows from the basic transversality theorems for maps of manifolds. If an equivariant map $f$ is constructed by equivariant transversality, then the information in $c$ is given fairly explicitly (compare [13]). More generally, the information carried by $c$ is needed to ensure that equivariant normal maps are characterizable by reasonable equivariant homotopy-theoretic invariants arising from equivariant transversality; abstract classifying spaces for these invariants may be produced using results of F. Connolly and V. Vijums [9]. Independent work of P. Löffler provides detailed classification information in an important special case (see [21], Korollar 2.8 , p. 297).

In many cases it is possible to construct $c$ from $f$ and $b$, and under a slight strengthening of the Gap Hypothesis there is an essentially unique way of doing so. We shall discuss these points in $\$ 4$. In contrast to this, there is no simple a priori condition for recovering $c$ in the analogous surgery theories for locally linear piecewise linear or topological actions. The reasons for this will be discussed at the end of $\S 4$. 
There is a natural notion of normal cobordism associated to the above definition of $\mathbf{Z}_{2}$-normal map; as in nonequivariant surgery, if two normal maps are related through a sequence of surgeries then the maps are normally cobordant. For the sake of completeness, a normal cobordism is defined to be a map of triads from $\left(W, X_{0}, X_{1}\right)$ to $Y \times[0,1]$ such that the restrictions to the $X_{l}$ are normal maps and the map on $W$ satisfies all the conditions for a normal map except perhaps the last.

We shall need a general position assumption that is close (but not identical) to the standard Gap Hypothesis. A connected smooth $\mathbf{Z}_{2}$-manifold $M$ will be said to satisfy the Borderline Gap Hypothesis provided the dimension of the fixed point set is at most $\frac{1}{2} \operatorname{dim} X$ and there is exactly one component of the fixed set whose dimension is actually equal to $\frac{1}{2} \operatorname{dim} X$. Such a component will be called a dominant component. For some results the existence of a dominant component is crucial (e.g., Theorem B).

The first step in $\mathbf{Z}_{2}$-surgery is to adjust the induced map of fixed point sets. By Smith theory, if the map is a pseudoequivalence then the induced map of fixed point sets must be a $\mathbf{Z}_{2}$-homology equivalence. Thus the first order of business is to see if one can modify $f$ to make the map of fixed point sets a $\mathbf{Z}_{2}$-homology equivalence. We shall say that a $\mathbf{Z}_{2}$-surgery problem is adjusted if the induced map of fixed point sets induces an isomorphism in $\mathbf{Z}_{2}$-homology. The problem of converting a normal map into an adjusted map is basically a question in nonequivariant surgery theory, and it can be answered using the homology surgery theory developed by S. Cappell and J. Shaneson [7]. Specifically, the discussion of $\S 2$ yields the following conclusion:

(1.2) Let $(f, b, c)$ be a $\mathbf{Z}_{2}$-normal map, let $F_{\alpha}$ denote the components of the fixed point sets of $Y$, let $n(\alpha)$ be the dimension of $F_{\alpha}$, and let $\pi_{\alpha}$ denote the fundamental group of $F_{\alpha}$. Then there are obstructions

$$
\sigma_{\alpha} \in \Gamma_{n(\alpha)}\left(\mathbf{Z}\left[\pi_{\alpha}\right] \rightarrow \mathbf{Z}_{2}\right)
$$

such that $(f, b, c)$ can be modified to an adjusted normal map if and only if all obstructions vanish.

The $\Gamma$-groups for homology surgery are those defined by Cappell and Shaneson in [7].

In some cases not all of the assumptions on $(f, b, c)$ are needed. Some specific comments in this direction appear in $\$ 2$. 
The second step in $\mathbf{Z}_{2}$-surgery involves modifications on the complement of the fixed point set (i.e., the set of free orbits). This process has been described in [11]; the surgery obstructions arising in that paper need to be reconsidered in order to accommodate the more general assumptions here. At this point it is important that there is one fixed point component of half the ambient dimension. There are two separate cases depending on whether $\operatorname{dim} X$ is congruent to 0 or $2 \bmod 4$.

Let $(f, b, c)$ be an adjusted $\mathbf{Z}_{2}$-normal map whose underlying degree 1 map is $f: X \rightarrow Y$. Denote the dominant components of the fixed point sets of $X$ and $Y$ by $X_{0}$ and $Y_{0}$ respectively. By assumption the induced map $f_{0}: X_{0} \rightarrow Y_{0}$ is a $\mathbf{Z}_{2}$-homology equivalence and consequently has an odd degree; this degree is well-defined up to sign and will be called $d$.

TheOREM $\mathbf{A}_{0}$. Let $(f, b, c)$ be an adjusted $4 k$-dimensional $\mathbf{Z}_{2}$-normal map as above. Assume that the Borderline Gap Hypothesis applies, both $X$ and $Y$ are without boundary, and that the dominant components of $X$ and $Y$ are orientable. Then $(f, b, c)$ is normally cobordant to a pseudoequivalence, relative to the fixed point sets, if and only if

(i) $\left[\operatorname{sgn}\left(\mathbf{Z}_{2}, X\right)-\operatorname{sgn}\left(\mathbf{Z}_{2}, Y\right)\right]=0$,

(ii) $\left(d^{2}-1\right) \operatorname{sgn}(T, Y)=0$.

Here $T$ represents the generator of $\mathbf{Z}_{2}$, and the equivariant signature invariants $\operatorname{sgn}(T, Y)$ and $\operatorname{sgn}\left(\mathbf{Z}_{2}, X\right.$ or $\left.Y\right)$ are defined in the standard fashion as in [11]. Theorem $A_{0}$ was stated and proved in [11] with a simple connectivity assumption on $X_{0}$ and $Y_{0}$.

If the dominant components of the fixed point sets are not orientable the conclusion is slightly different.

TheOREM $\mathrm{A}_{2}$. Let $(f, b, c)$ be an adjusted $4 k$-dimensional $\mathbf{Z}_{2}$-normal map as in the preceding theorem, but assume that the dominant components of $X$ and $Y$ are not orientable and drop the assumption that $X$ and $Y$ are without boundary. Then $(f, b, c)$ is normally cobordant to a pseudoequivalence, relative to the fixed point sets and boundaries, if and only if $\left[\operatorname{sgn}\left(\mathbf{Z}_{2}, X\right)-\operatorname{sgn}\left(\mathbf{Z}_{2}, Y\right)\right]=0$.

Special cases of this result were mentioned and used in [12] and [19].

We shall now consider adjusted $\mathbf{Z}_{2}$-normal maps in the $(4 k+2)$ dimensional case. Two invariants are relevant. The first is the Arf invariant $\mathbf{C}(f, b, c)$ of the ordinary normal map obtained by forgetting the 
involution. To describe the second invariant, make $f$ connected up to the middle dimension by surgery on the free part. As in [11] it follows that the only nonvanishing surgery kernel $K_{2 k+1}(Y)$ is free over the group ring $\mathbf{Z}\left[\mathbf{Z}_{2}\right]$. Take the rank of this free module over $\mathbf{Z}\left[\mathbf{Z}_{2}\right]$ and reduce it mod 2 . This invariant is called $\mathbf{r}(f, b, c)$, and it turns out to depend only on the normal cobordism class of the normal map (compare [11], Lemma 3.1, page 277).

Theorem B. Let $(f, b, c)$ be an adjusted $(4 k+2)$-dimensional $\mathbf{Z}_{2}$-normal map, and assume that the Borderline Gap Hypothesis holds. Then $(f, b, c)$ is normally cobordant to a pseudoequivalence, relative to the fixed point sets and boundaries, if and only if $\mathbf{C}(f, b, c)$ and $\mathbf{r}(f, b, c)$ are both zero.

The proofs of Theorems $\mathrm{A}_{0}, \mathrm{~A}_{2}$, and $\mathrm{B}$ will be discussed in $\S 3$.

One can also attempt to modify a normal map to an equivariant homotopy equivalence rather than merely a pseudoequivalence. In this case each of the induced maps on fixed point components must be converted into a homotopy equivalence. Consequently, one needs an additional assumption that the degree of each induced map be \pm 1 . Furthermore, we must also assume that the map on the boundaries is an equivariant homotopy equivalence. A normal map that satisfies these restrictions will be called a normal map of type $\mathbf{h}$ or an $\mathbf{h}$-normal map. For normal maps of type $\mathbf{h}$ we have the following conclusion:

THeOREM C. Let $(f, b, c)$ be a 2 m-dimensional $\mathbf{Z}_{2}$-normal map of type $\mathbf{h}$ satisfying the Borderline Gap Hypothesis. If $m$ is even assume both $X$ and $Y$ are without boundary. Then $(f, b, c)$ is $\mathbf{h}$-normally cobordant to a $\mathbf{Z}_{2}$-homotopy equivalence, relative to the fixed point sets and boundaries, if and only if

(i) all surgery obstructions $\boldsymbol{\sigma}_{\alpha}^{h}\left(f_{\alpha}\right) \in L_{n(\alpha)}^{h}\left(\mathbf{Z}\left[\pi_{\alpha}\right], w_{\alpha}\right)$ are zero,

(ii) $\left[\operatorname{sgn}\left(\mathbf{Z}_{2}, X\right)-\operatorname{sgn}\left(\mathbf{Z}_{2}, Y\right)\right]=0$ if $m$ is even,

(iii) $\mathbf{C}(f, b, c)$ and $\mathbf{r}(f, b, c)$ are both zero if $m$ is odd.

In $\S 3$ we shall indicate how one can modify the proofs of the preceding results to establish this theorem. The notation in part (i) is parallel to that of (1.2), with $w_{\alpha}$ denoting the first Stiefel-Whitney class of $F_{\alpha}, L^{h}$ the obstruction group for surgery up to homotopy equivalence, and $\sigma^{h}$ the algebraic obstruction for surgery up to homotopy equivalence. 
Similarly, one can attempt to modify a normal map to a simple equivariant homotopy equivalence in the sense of [17] or [27] rather than merely an equivariant homotopy equivalence. Equivariant versions of the $s$-cobordism theorem (e.g., see [14] or [27]) show that simple $\mathbf{Z}_{2}$-surgery is very closely related to the classification of smooth $\mathbf{Z}_{2}$-manifolds up to equivariant diffeomorphism. The appropriate notion of normal map in this case (i.e., an s-normal map) requires still further assumptions. Specifically, we must also assume the map on the boundaries is a simple equivariant homotopy equivalence. In this case we have the following analog of Theorem C:

THEOREM $\mathbf{C}_{\mathbf{s}}$. Let $(f, b, c)$ be a 2 m-dimensional $\mathbf{Z}_{2}$-normal map of type $\mathbf{s}$ satisfying the Borderline Gap Hypothesis. If $m$ is even assume both $X$ and $Y$ are without boundary. Then $(f, b, c)$ is $\mathbf{s}$-normally cobordant to a $\mathbf{Z}_{2}$-simple homotopy equivalence, relative to the fixed point sets and boundaries, if and only if

(i) all simple surgery obstructions $\sigma_{\alpha}^{s}\left(f_{\alpha}\right) \in L_{n(\alpha)}^{s}\left(\mathbf{Z}\left[\pi_{\alpha}\right], w_{\alpha}\right)$ are zero,

(ii) the appropriate condition (ii) or (iii) in the precedisng theorem is fulfilled.

Notation is basically the same as in Theorem $\mathrm{C}$, with $L^{s}$ denoting the obstruction group for surgery up to simple homotopy equivalence and $\sigma^{s}$ the algebraic obstruction for surgery up to simple homotopy equivalence.

The results above were stated in simplified forms in order to keep the discussion relatively uncluttered. In many cases it is possible to obtain similar results with weaker data. For example, we could consider normal maps whose codomains are $\mathbf{Z}_{2}$-Poincaré duality complexes. In particular, Theorems $\mathrm{A}_{2}, \mathrm{~B}$, and $\mathrm{C}$ generalize. However, Theorem $\mathrm{A}_{0}$ uses and Atiyah-Singer $G$-signature Theorem and thus requires that $Y$ be smooth.

\section{Comparisons to other equivariant surgery settings}

It is instructive to compare the preceding results with the corresponding conclusions under the Gap Hypothesis. The obstructions to surgery on the fixed point set remain the same, but under the Gap Hypothesis the surgery obstruction for an adjusted normal map lies in a Wall group $L_{2 n}\left(\mathbf{Z}\left[\mathbf{Z}_{2}\right], w\right)$, where $w$ is trivial or nontrivial depending on whether the involution preserves or reverses orientation. This result is contained in many treatments of equivariant surgery theory including [13], [14], and [21]. 
Much earlier work of W. Browder and F.Quinn yields a setting for equivariant surgery in which the Gap Hypothesis need not be assumed but the normal maps must satisfy some other restrictions [6]. In particular, it is meaningful to apply the Browder-Quinn theory in the setting of this paper, and in fact one can show that, within this setting, every BrowderQuinn surgery problem gives rise to a surgery problem as defined in (1.1a-d) above (compare [15]). It follows that every Browder-Quinn surgery obstruction determines a well-defined surgery obstruction given by one of the theorems stated above. The relationship between these surgery obstructions is discussed in [11] (in particular, see Example 4.13, page 281).

2. Adjusting maps of fixed point sets. As noted in $\$ 1$, the first step in $\mathbf{Z}_{2}$-surgery is an attempt to make the map of fixed point sets into an equivalence of the appropriate type. This type depends on whether we are considering surgery up to pseudoequivalence, $\mathbf{Z}_{2}$-homotopy equivalence, or simple $\mathbf{Z}_{2}$-homotopy equivalence. Although the discussions differ in details, the overall patterns are similar. In particular, the initial portion is identical in all three cases.

Let $(f, b, c)$ be a $\mathbf{Z}_{2}$-normal map with $f: X \rightarrow Y$. As in $\S 1$ the underlying components of the fixed point set of $Y$ will be denoted by $F_{\alpha}$ and the induced map of fixed point sets will be written as a disjoint union of maps $\dot{f}_{\alpha}: E_{\alpha} \rightarrow F_{\alpha}$. The conditions on a normal map imply that each $E_{\alpha}$ is nonempty and of the same dimension as $F_{\alpha}$; this dimension is denoted by $n(\alpha)$.

If $b: T X \rightarrow f^{*} \xi$ is the $\mathbf{Z}_{2}$-vector bundle map for the given $\mathbf{Z}_{2}$-surgery problem, then as noted in $\S 1$ there is a splitting of $b \mid E_{\alpha}$ into $b_{\alpha}(+) \oplus$ $b_{\alpha}(-)$ corresponding to the eigenspaces of \pm 1 . The pair $\left(f_{\alpha}, b_{\alpha}(+)\right)$ is then a normal map in the usual nonequivariant sense. By assumption its degree $d_{\alpha}$ is \pm 1 for surgery up to $\mathbf{Z}_{2}$-homotopy equivalence or simple $\mathbf{Z}_{2}$-homotopy equivalence, and the degree is odd for surgery up to pseudoequivalence (compare [4]).

The study of the map of fixed point sets splits naturally into the study of the individual normal maps $\left(f_{\alpha}, b_{\alpha}(+)\right)$ and therefore when convenient we shall concentrate on some fixed but arbitrary component $F_{\alpha}$.

At this point the discussion separates into the three individual cases. Since the treatments for surgery up to $\mathbf{Z}_{2}$-homotopy equivalence (both ordinary and simple) are the most standard, we shall consider them first.

For surgery up to (ordinary or simple) $\mathbf{Z}_{2}$-homotopy equivalence, the goal is to modify the map of fixed point sets into an ordinary or simple homotopy equivalence respectively. The fundamental results of surgery 
theory imply this is possible if certain surgery obstructions

$$
\boldsymbol{\sigma}_{\alpha}^{B}\left(f_{\alpha}, b_{\alpha}(+)\right) \in L_{n(\alpha)}^{B}\left(\mathbf{Z}\left[\pi_{\alpha}\right], w_{\alpha}\right)
$$

are zero, where $B=h$ for homotopy equivalence and $B=s$ for simple homotopy equivalence. Strictly speaking, we must have $n(\alpha) \geq 5$ to apply surgery theory. However, our definitions stipulate that $f_{\alpha}$ be an ordinary or simple homotopy equivalence in dimensions less than 5 , and consequently the conclusion in lower dimensions is trivially true by our choice of definition.

For surgery up to pseudoequivalence, the goal is to modify the map of fixed point sets into a $\mathbf{Z}_{2}$-homology equivalence. If the degree $d_{\alpha}$ of $f_{\alpha}$ is \pm 1 , then results of Cappell and Shaneson [7] state that $\left(f_{\alpha}, b_{\alpha}(+)\right)$ can be modified to a $\mathbf{Z}_{2}$-homology equivalence if and only if an algebraic surgery obstruction

$$
\sigma_{\alpha}^{\mathbf{F}}\left(f_{\alpha}, b_{\alpha}(+)\right) \in \Gamma_{n(\alpha)}\left(\mathbf{F}: \mathbf{Z}\left[\pi_{\alpha}\right] \rightarrow \mathbf{Z}_{2}\right)
$$

vanishes. The appropriate homomorphism from $\mathbf{Z}\left[\pi_{\alpha}\right]$ to $\mathbf{Z}_{2}$ is the augmentation sending each group element to 1 . Definitions of the $\Gamma$-groups may be found in [7], $\S \S 1$ and 2 (especially pages $285-288$ and following Proposition 2.1 on page 296).

In order to treat maps $f_{\alpha}$ whose degrees $d_{\alpha}$ are odd integers but not \pm 1 , we need the following observation:

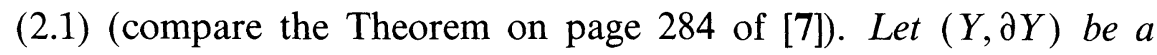
manifold pair with $\pi_{1}(Y)=\pi$ and $n=\operatorname{dim} Y \geq 5$. Let $\Lambda$ be a ring with involution and

$$
\mathbf{F}: \mathbf{Z}[\pi] \rightarrow \Lambda
$$

a local epimorphism of rings with involution. Let $(f, b)$ be a normal map into $(Y, \partial Y)$ such that the degree $d$ is a unit in $\Lambda$ and the induced map on the boundary is a $\Lambda$-homology equivalence. Then $(f, b)$ determines an element $\sigma(f, b) \in \Gamma_{n}(F)$, and this element vanishes if and only if $(f, b)$ is normally cobordant, relative the boundary, to a $\Lambda$-homology equivalence.

The proof of this result is nearly identical to the proof in [7] for the case $d_{\alpha}= \pm 1$. Of course, a few additional remarks are needed. There is no problem making the domain connected; this can be done by adding 1-handles to form connected sums exactly as in the degree 1 case. On the other hand, the usual arguments for making a map 1-connected require some modifications. Degree 1 maps of connected manifolds always induce surjections of fundamental groups, but the corresponding statement for 
maps of higher degree is generally false. However, even in these cases one can perform low-dimensional surgery to make the domain connected and the map of fundamental groups bijective (compare [15]). Let $C_{d}$ denote the Serre class of abelian groups whose tensor products with $\mathbf{Z}\left[d^{-1}\right]$ are trivial. One can then proceed to make the normal map highly connected mod $C_{d}$ as in the first three sections of [7], the main change being the need to use the $\bmod C_{d}$ relative Hurewicz Theorem rather than the standard relative Hurewicz Theorem. The algebra involving $\Lambda$-homology becomes formally the same as in [7] provided the degree $d$ is a unit in $\Lambda$; for example, the relative homology with local coefficients in $\Lambda$ vanishes except in the middle dimension(s). The middle dimensional surgery kernels with coefficients in $\Lambda$ may now be studied exactly as in [7]. One easy way to see this (suggested by S. Weinberger) is to replace the fundamental class of $(Y, \partial Y)$ with $d^{-1}$ times the usual fundamental class. With this convention the given degree $d$ normal map $f$ induces a degree 1 map in $\Lambda$-homology and the discussion in the first chapter of [7] applies word for word.

As in the other cases, one cannot apply the results and methods of [7] directly if $n(\alpha) \leq 4$, but on the other hand our definitions require that $f_{\alpha}$ be a homology equivalence in these dimensions, and consequently the conclusion in lower dimensions is once again a tautology by our choice of definition.

It now follows that the induced map of fixed point sets can be converted into an equivalence of the appropriate type if and only if the corresponding surgery obstructions $\sigma_{\alpha}$ are all zero. However, we need something stronger; namely, the $\mathbf{Z}_{2}$-normal map $(f, b, c)$ is $\mathbf{Z}_{2}$-normally cobordant to an adjusted map if and only if the obstructions $\sigma_{\alpha}$ are all zero. This will be a consequence of the preceding discussion and the following normal cobordism extension theorem:

THEOREM 2.2. Let $(f, b, c)$ be a $\mathbf{Z}_{2}$-normal map with domain $X$, and let $\left(F^{\prime}, B^{\prime}\right)$ be a disjoint union of normal cobordisms with domain $V$ and $\partial_{0} F^{\prime}=\operatorname{Fix}(f)$. Then there is a $\mathbf{Z}_{2}$-normal cobordism $(F, B, C)$ such that $\partial_{0} F=f$ and $\operatorname{Fix}(F)=F^{\prime}$.

Derivation of (1.2) from Theorem 2.2. Suppose that the $\sigma_{\alpha}$ all vanish. Then one can construct a map $F^{\prime}$ as above with $\partial_{1} F^{\prime}$ an equivalence. Take $F$ to be an extension of $F^{\prime}$ as given by Theorem 2.2. Then $F$ defines a $\mathbf{Z}_{2}$-normal cobordism from $f$ to an adjusted $\mathbf{Z}_{2}$-normal map $\partial_{1} F$.

Proof of 2.2. The basic idea is fairly standard (compare Browder [4], pp. 716-717). Let $V$ denote the cobordism on which $F^{\prime}$ is defined. 
Observe that the equivariant normal bundle of $\partial_{0} V$ in $X$ is identified with $f^{*} \eta$ via $c$, and this vector bundle extends to a vector bundle $\omega=F^{* *} \eta$ over $V$. If we denote the disk and sphere bundles for a vector bundle $\gamma$ by $D(\gamma)$ and $S(\gamma)$ respectively, then it follows that

$$
\partial D(\omega)=S(\omega) \cup D\left(\omega \mid \partial_{0} V\right) \cup D\left(\omega \mid \partial_{1} V\right) .
$$

By construction $\omega_{0}=\omega \mid \partial_{0} V$ is the normal bundle $f^{*} \eta$.

From the locally linear $\mathbf{Z}_{2}$-manifold

$$
W_{0}=X \times[-1,0] \cup D(\omega),
$$

where $D\left(\omega_{0}\right) \subseteq \partial D(\omega)$ is identified (equivariantly!) with a closed tubular neighborhood of $\partial_{0} V$ in $X \times\{0\}$ via $c$. The manifold $W_{0}$ is in fact a smooth $\mathbf{Z}_{2}$-manifold with corners, and by a standard argument as in [10] these corners may be rounded to produce a smooth approximation $W$ that is piecewise differentiably isomorphic to $W_{0}$ (see Figure 1 below).

Since $X \times\{0\} \cup V$ is an equivariant strong deformation retract of $W_{0}$ and $W$, it is fairly straightforward to extend the data

$$
(f, b, c) \cup\left(F^{\prime}, B^{\prime}\right)
$$

to a $\mathbf{Z}_{2}$-normal bordism $(F, B, C)$. The upper end of this bordism has domain

$$
\left(X \times\{0\}-\operatorname{Int} D\left(\omega_{0}\right)\right) \cup S(\omega) \cup D\left(\omega \mid \partial_{1} V\right)
$$

and fixed point set $\partial_{1} V$. By construction the restriction of the bordism to the fixed point set is precisely $F^{\prime}$.

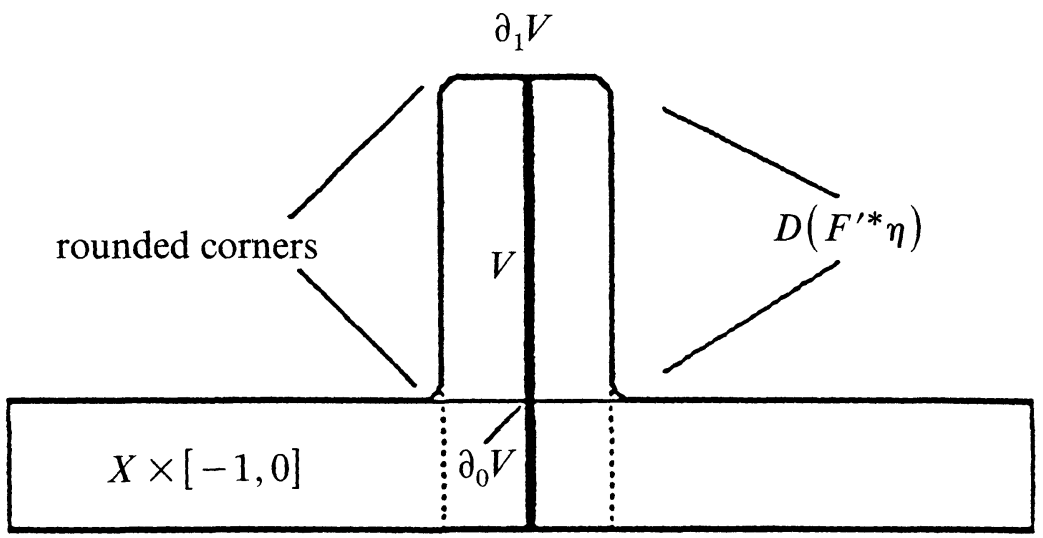




\section{Relative version of (1.2)}

In some cases it is possible to do without some of the usual data. For example, suppose there is a set $\mathbf{B}$ of components $F_{\beta}$ such that each map $f_{\beta}$ is an equivalence of the desired type. Then it is not necessary to have the unstable bundle data $c_{\beta}$ for $\beta \in \mathbf{B}$. In this case one still has surgery obstructions $\sigma_{\alpha}$ for $\alpha$ not in $\mathbf{B}$, and a normal map is normally cobordant to an adjusted map, relative to the boundaries and the components in $\mathbf{B}$, if and only if these obstructions vanish.

3. Surgery on the free part. Once a $\mathbf{Z}_{2}$-normal map has been modified to be an equivalence on the fixed point set, the next step is to attempt surgery on the complement (i.e., the free part of the $\mathbf{Z}_{2}$-manifold) such that the original map on the fixed point sets is left unchanged. Throughout this section we assume that the Borderline Gap Hypothesis holds.

Let $(f, b, c)$ be an adjusted $\mathbf{Z}_{2}$-normal map. One can do surgery below the middle dimension exactly as in [11] to make $f$ connected up to the middle dimension of the underlying manifolds $X$ and $Y$. If this dimension is $2 m$, then the only surgery kernel that can be nonzero is in dimension $m$, and this kernel is a free $\mathbf{Z}\left[\mathbf{Z}_{2}\right]$-module.

The kernel $K_{m}$ has two associated forms; namely, the $(-1)^{m}$-Hermitian intersection form $\lambda$ and the quadratic self-intersection form $\mu$. The basic algebraic properties of the quasi-Hermitian form $(K, \lambda, \mu)$ are listed on pages 268-269 of [11]; these are similar to the properties of a Hermitian form as defined by Wall [30], but the associated form $x \cap T y$ over the integers need not be even. The results of [11] show that the Witt classes of these forms are completely characterized by

(i) the equivariant signature if $m$ is even (see [11], Theorem 2.3, page 274),

(ii) the Arf invariant and the mod 2 rank over the group ring if $m$ is odd (see [11], Theorem 4.3, page 281).

A quasi-Hermitian form represents the zero Witt class if and only if there is a free submodule $N$ such that $\lambda$ and $\mu$ both vanish on $N$ and furthermore $N$ is its own orthogonal complement. Following Wall [30] we shall call such a submodule a subkernel.

Suppose now that $\left(K_{m}, \lambda, \mu\right)$ represents the zero Witt class. The standard techniques of surgery theory allow us to represent a basis of the free submodule $N$ by embedded spheres. One would like to do surgery on these classes in order to kill $K_{m}$ as in [28]. However, in order to do this we must know that the embedded spheres miss the fixed point set of $X$. The results of [11] show that one can find such embedded spheres if the 
dominant components of the fixed point sets are orientable. This immediately yields Theorem $\mathrm{A}_{0}$ and also Theorem $\mathrm{B}$ when the dominant components of the fixed point sets are orientable. The following well-known version of the Whitney trick supplies the information needed when the dominant components are not orientable.

LEMMA 3.1. Let $X$ be a simply connected $2 m$-dimensional manifold and $F$ an $m$-dimensional nonorientable submanifold of $X$, where $m \geq 3$. Let $j$ be an embedding of $S^{m}$ in $X$ intersecting $F$ transversely. Then the embedding $j$ is regularly homotopic to an embedding $j_{1}$ disjoint from $F$ if and only if the image of $j$ intersects $F$ in an even number of points.

Let $x \in K_{m}$ be represented by an embedding $j$ of $S^{m}$. It is easy to compute the mod 2 number of intersection points in $j\left(S^{m}\right) \cap F$. Let $T$ be the generator for $\mathbf{Z}_{2}$, and approximate $j$ by a smooth embedding that is also transverse to $T_{j}$. Away from the fixed point set the intersection points for $j\left(S^{m}\right)$ and $T j\left(S^{m}\right)$ appear in pairs, and thus we obtain the following conclusion:

(3.2) The mod 2 intersection number of $F$ and $j\left(S^{m}\right)$ is given by $\lambda(x, T x) \bmod 2$.

Proofs of Theorems $\mathrm{A}_{2}$ and $\mathrm{B}$ (nonorientable case).

In either case the geometric information implies that the Witt class of the quasi-Hermitian form $\left(K_{m}, \lambda, \mu\right)$ is zero. Thus we have an algebraic subkernel $N$ inside $K_{m}$. Choose a free basis of $N$, say $\left\{e_{1}, \ldots, e_{k}\right\}$, and represent the $e_{i}$ by smooth embeddings $g_{i}$. Since $\lambda\left(e_{i}, T e_{i}\right)=0$, the preceding discussion implies that the $g_{i}$ may be chosen so that their images are disjoint from $F$. The conditions on $N$ also imply that the images of $g_{i}$ and $g_{j}$ may be chosen to be disjoint if $i \neq j$, and furthermore the images of $g_{i}$ and $T g_{j}$ may be chosen to be disjoint for all $i$ and $j$. Therefore we may apply the standard techniques of surgery theory to the embeddings $g_{i}$ and their translates under $T$; if one performs surgery equivariantly along these classes, the standard results of surgery theory imply that the modified map has zero surgery kernel in the middle dimension and is therefore a homotopy equivalence.

\section{Proofs of Theorems $\mathrm{C}$ and $\mathrm{C}_{\mathrm{s}}$.}

The argument for Theorem $\mathrm{C}$ is similar to the proofs of Theorems $\mathrm{A}_{0}$, $\mathrm{A}_{2}$, and B. By Theorem 2.2 an $\mathbf{h}$-normal map is equivalent to an h-adjusted map if and only if the homotopy surgery obstructions of the $\int_{\alpha}$ all vanish. The preceding discussion of adjusted maps dealt exclusively 
with surgery steps that are disjoint from the fixed point set, and therefore the vanishing of these obstructions is sufficient to modify an adjusted h-normal map so that it induces homotopy equivalences on both the fixed point sets and the ambient $\mathbf{Z}_{2}$-manifolds. Since the basic results in equivariant homotopy theory imply that such a map is an equivariant homotopy equivalence (compare [3], Corollary 5.5, p. II.12), this concludes the proof.

The same sort of argument also proves Theorem $\mathrm{C}_{\mathrm{s}}$. By Theorem 2.2 an s-normal map is equivalent to an s-adjusted map if and only if the simple homotopy surgery obstructions of the $f_{\alpha}$ all vanish. As in the proof of Theorem $\mathrm{C}$, the vanishing conditions are sufficient to modify an adjusted s-normal map so that it induces simple homotopy equivalences on the fixed point set components and a pseudoequivalence on the ambient $\mathbf{Z}_{2}$-manifolds. Since the Whitehead group of $\mathbf{Z}_{2}$ is trivial, the modified map is a simple equivariant homotopy equivalence by the standard algebraic splitting of equivariant Whitehead groups (compare [17] and [27]).

4. Unstable data and the borderline gap hypothesis. In [11] the definition of a $\mathbf{Z}_{2}$-normal map does not include the unstable data given by c. It is also possible to prove versions of the main results of this paper without using $c$ if either the Gap Hypothesis or the Borderline Gap Hypothesis holds. An examination of $\$ \S 1-3$ shows that $c$ is only needed in the proof of Theorem 2.2. The argument in [11], paragraph 1.1, page 272 , provides an alternate approach that yields (1.2) of this paper without using $c$ or Theorem 2.2. Thus it is natural to ask if the information carried by $c$ is redundant. In this section we shall show that a degree 1 map $f$ and a stable bundle map $b$ almost always yield a choice for the unstable data $c$ under the Gap Hypothesis or Borderline Gap Hypothesis (see Theorem 4.2 below); furthermore, this choice is almost always unique if the Gap Hypothesis holds. In fact, the equivariant surgery obstructions only depend on a portion of the unstable data, and following Dovermann and Rothenberg [14] we formalize this by defining unstable data with deficiency. Under the Gap Hypothesis or the Borderline Gap Hypothesis there is a unique choice of unstable data with deficiency, and this fact is implicit in [11], paragraph 1.1, page 272.

Although the information carried by unstable data is redundant for many of our purposes, it seems worthwhile to retain the extra structure for several reasons. First, one needs unstable data to define a notion of 
equivariant normal map that has a purely homotopy-theoretic interpretation (compare the comments in $\$ 1$ ). Second, suitable unstable data cannot always be found if the Gap Hypothesis and the Bounded Gap Hypothesis fail to hold, and the same applies for unstable data with deficiency. Further analyses of $\mathbf{Z}_{2}$-surgery obstructions without such hypotheses will almost certainly rely more heavily on the homotopy-theoretic invariants of $\mathbf{Z}_{2}$-normal maps, and therefore it seems highly advisable to retain the unstable data $c$ for the sake of uniformity. Additional reasons for discussing unstable data explicitly are mentioned at the end of this section.

We shall begin by considering the existence and uniqueness of unstable data $c$ associated to a map $f$ and stable bundle data $b$. Both the existence of a choice for $c$ and the classification of all possible choices reduce to standard questions in homotopy theory. In order to state the basic results, we need a substantial amount of notation. As in $\S 1$, let $f$ : $X \rightarrow Y$ be an equivariant map between two smooth $\mathbf{Z}_{2}$-manifolds, $b$ : $T X \rightarrow f^{*} \xi$ a vector bundle isomorphism covering the identity on $X, F_{\alpha}$ the components of the fixed point sets of $Y$, and $E_{\alpha}$ the union of all components of the fixed point set of $X$ that are mapped into $F_{\alpha}$ by $f$. The map induced by $f$ from $E_{\alpha}$ to $F_{\alpha}$ will be called $f_{\alpha}$. Furthermore, split the restriction $b_{\alpha}$ of $b$ to $E_{\alpha}$ into a direct sum $b_{\alpha}(+) \oplus b_{\alpha}(-)$, corresponding to the eigenspaces of \pm 1 , and define $N E_{\alpha}$ to be the normal bundle of $E_{\alpha}$ in $X$. If $\gamma$ is an $m$-dimensional vector bundle over a space $B$, let $\operatorname{Prin}(\gamma)$ denote the associated principal bundle of orthonormal $m$-frames with respect to some Riemannian metric.

Let $q(\alpha)$ be the dimension of $N E_{\alpha}$, and let $q(\alpha)+s(\alpha)$ denote the fiber dimension for the domain and codomain of $b_{\alpha}(-)$. It follows that $b_{\alpha}(-)$ induces an $O_{q(\alpha)+s(\alpha)}$-equivariant map of principal bundles that we shall call $B_{\alpha}(-)$. There is a natural inclusion of $\operatorname{Prin}\left(N E_{\alpha}\right)$ in $\operatorname{Prin}\left(N E_{\alpha} \oplus \mathbf{R}^{s}\right)$ given by sending a $q$-frame $V=\left(v_{1}, \ldots, v_{q}\right)$ to $\left(v_{1}, \ldots, v_{q} ; e_{1}, \ldots, e_{s}\right)$ for some orthonormal basis $\left(e_{1}, \ldots, e_{s}\right)$ of $\mathbf{R}^{s}$. This map is equivariant with respect to the natural action of $O_{q(\alpha)}$ on the domain and the action on the codomain induced by restriction to the standardly embedded copy of $O_{q(\alpha)}$ in $O_{q(\alpha)+s(\alpha)}$. Let $B_{\alpha}^{\prime}$ denote the restriction of $B_{\alpha}(-)$ to $\operatorname{Prin}\left(N E_{\alpha}\right)$. Standard theorems on equivariant maps (compare Cartan [8], Heller [16]) imply that $B_{\alpha}(-)$ corresponds to a lifting $B_{\alpha}^{*}$ of the associated fiber bundle

$$
\psi_{\alpha}: \operatorname{Prin}\left(\xi_{\alpha}(-)\right) \times_{O(q(\alpha))} \operatorname{Prin}\left(N E_{\alpha}\right) \rightarrow F_{\alpha} .
$$


Let $\psi_{\alpha}^{*}$ be the induced bundle of $\psi_{\alpha}$ under the canonical projection from the mapping cylinder of $f_{\alpha}$ to $F_{\alpha}$. It follows that $B_{\alpha}^{*}$ determines a section of $\psi_{\alpha}^{*}$ restricted to $E_{\alpha}$ viewed as a subspace of the mapping cylinder $\operatorname{Cyl}\left(f_{\alpha}\right)$.

Let

$$
\rho_{\alpha}: \operatorname{Prin}\left(\xi_{\alpha}(-)\right) \times_{O(q(\alpha))} \operatorname{Prin}\left(N E_{\alpha}\right) \rightarrow \operatorname{Prin}\left(\xi_{\alpha}(+)\right) / O(q(\alpha))
$$

be the canonical map induced by projection onto the first factor, and let

$$
\omega_{\alpha}: \operatorname{Prin}\left(\xi_{\alpha}(-)\right) / O(q(\alpha)) \rightarrow F_{\alpha}
$$

be the fiber bundle projection determined by the principal $O_{q(\alpha)+s(\alpha)}$-bundle. Observe that the fiber of this bundle is the Stiefel manifold $O_{q(\alpha)+s(\alpha)} / O_{q(\alpha)}$. In analogy with the preceding paragraph let $\omega_{\alpha}^{*}$ be the induced bundle of $\omega_{\alpha}$ under the canonical projection from the mapping cylinder of $f_{\alpha}$ to $F_{\alpha}$. It then follows that the composite map $\rho_{\alpha} B_{\alpha}^{*}$ determines a section of $\omega_{\alpha}^{*}$ restricted to $E_{\alpha}$ viewed as a subspace of the mapping cylinder $\operatorname{Cyl}\left(f_{\alpha}\right)$.

We can now state the homotopy-theoretic criteria for the existence of the unstable data $c_{\alpha}$ over some arbitrary $F_{\alpha}$ and also the classification of all such choices up to equivalence.

Proposition 4.1. In the notation developed above, there is a 1-1 correspondence between equivalence classes of pairs $\left(\dot{c}_{\alpha}, \ddot{c}_{\alpha}\right)$ as in the definition of $\mathbf{Z}_{2}$-normal map and relative homotopy classes of cross sections of $\omega_{\alpha}^{*}$ that agree with $\rho_{\alpha} B_{\alpha}^{*}$ on $E_{\alpha} \subseteq \operatorname{Cyl}\left(f_{\alpha}\right)$.

This result is a routine consequence of standard results on the reducibility of the structure group of a principal $O_{q+s}$-bundle to an $O_{q}$-bundle (once again see Cartan [8] or Heller [16]).

It follows that one can define homotopy-theoretic obstructions to the existence of $c_{\alpha}$ with values in the cohomology groups

$$
H^{\prime}\left(\operatorname{Cyl}\left(f_{\alpha}\right), E_{\alpha} ; \pi_{l-1}\left(O_{q(\alpha)+s(\alpha)} / O_{q(\alpha)}\right)\right) ;
$$

in general these cohomology groups involve twisted coefficients (as in all obstruction-theoretic problems). Given two choices $c_{\alpha}$ and $c_{\alpha}^{\prime}$ one has corresponding obstructions to the equivalence of these choices with coefficients in $\pi_{l}$ rather than $\pi_{i-1}$. These lead to the following sufficient conditions for the existence and uniqueness of $c_{\alpha}$ :

Proposition 4.2. Let $f: X \rightarrow Y, b: T X \rightarrow f^{*} \xi, F_{\alpha}, E_{\alpha}, f_{\alpha}$ be as described in the second paragraph of this section. Denote the dimensions of $Y$ and $F_{\alpha}$ by $m$ and $n(\alpha)$ respectively. 
(i) If $n(\alpha)<\frac{1}{2} m$ then one can find unstable data $c_{\alpha}$ over the component $F_{\alpha}$, and if $n(\alpha)<\frac{1}{2}(m-1)$ there is a unique choice for such data up to equivalence.

(ii) If $n(\alpha)=\frac{1}{2} m$ then one can find unstable data $c_{\alpha}$ over the component $F_{\alpha}$ under any of the following conditions:

(a) $m$ is congruent to 2 mod 4.

(b) $F_{\alpha}$ is nonorientable.

(c) The degree of $f_{\alpha}$ is 1 .

In each of the cases described above, the obstructions lie in cohomology groups that are automatically zero. It follows immediately that one can always find unstable data $c_{\alpha}$ whenever the Gap Hypothesis holds.

If the Borderline Gap Hypothesis holds, then Proposition 4.2 implies that unstable bundle data can be found over all fixed point components except perhaps the dominant component. Furthermore, data can also be found for the latter except perhaps when $m$ is divisible by 4 , the dominant component is orientable, and the degree of the map of dominant components has absolulte value greater than 1 . In particular, one can always find unstable data in the settings of Theorems $\mathrm{A}_{0}, \mathrm{~A}_{2}, \mathrm{~B}, \mathrm{C}$, and $\mathrm{C}_{0}$. On the other hand, even if none of conditions (a)-(c) is valid, it is still possible to describe the obstruction to the existence of unstable data over the dominant component very simply.

Proposition 4.3. In the preceding notation, assume that the Borderline Gap Hypothesis holds but conditions $(a)-(c)$ above do not hold. Then unstable data $c_{\alpha}$ can be constructed over the dominant component if and only if the Euler class of the normal bundle $N E_{\alpha}$ is divisible by the degree $d_{\alpha}$ of the $\operatorname{map} f_{\alpha}$.

The necessity of this condition is immediate, for if $c_{\alpha}$ exists then $N E_{\alpha}$ is the pullback of some bundle under $f_{\alpha}$. To see sufficiency, use the fact that the Euler class defines the primary obstruction to lifting from BSO to $\mathrm{BSO}_{2 k}$.

\section{Unstable data and values of surgery obstructions}

In general there are several choices of $c$ that make $(f, b, c)$ into a normal map. If either the Gap Hypothesis or the Borderline Gap Hypothesis holds, one can use the methods of James and Thomas [18], Sutherland [29], or Becker [2] to find reasonable homotopy-theoretic 
invariants that classify the different choices up to equivalence. It is natural to ask if the equivariant surgery obstructions depend upon the choice of $c$. In the remainder of this section we shall develop machinery to show that the obstructions are in fact independent of $c$ provided the Gap Hypothesis or the Borderline Gap Hypothesis holds. The key step is to define a slight weakening of unstable bundle data.

\section{Unstable data with deficiencies}

We wish to consider unstable data that is defined on only a portion of the fixed point set. In order to do this we need to describe the sorts of subsets on which the unstable data need not be defined. First, we shall only consider closed $\mathbf{Z}_{2}$-invariant subsets of locally linear $\mathbf{Z}_{2}$-manifolds that are unions of locally linear $\mathbf{Z}_{2}$-submanifolds and whose intersections with all components of the fixed point sets are submanifolds. If the ambient manifold has a boundary, we assume the submanifolds are properly embedded (boundary to boundary and interior to interior). Such a union of submanifolds will be called a geometric $\mathbf{Z}_{2}$-variety. Given a locally linear $\mathbf{Z}_{2}$-manifold $M$ without boundary, we shall say that a geometric $\mathbf{Z}_{2}$-variety $P$ is saturating (or saturates $M$ ) if for each compact component $C$ of the fixed point set the intersection $P \cap C$ is nonempty. We shall say that $P$ is $k$-saturating if in addition the inclusion of $P \subseteq C$ is $k$-connected (hence saturating means 0 -saturating).

Let $f: X \rightarrow Y$ be a degree 1 map and let $b$ be stable bundle data for $f$ as in the preceding discussion. Following the ideas of [14], we define unstable data with deficiency $k$ to consist of a $k$-saturating geometric variety $P_{X} \subseteq X$ with dimension at most $k$, a $(k-1)$-saturating geometric variety $R_{Y} \subseteq Y$ with dimension at most $(k-1)$, a family of vector bundles $\eta_{\alpha}$ over the $F_{\alpha}-R_{Y}$, and vector bundle isomorphisms $c_{\alpha}=$ $\left(\dot{c}_{\alpha}, \ddot{c}_{\alpha}\right)$ defined over the sets $E_{\alpha}-P_{X}$. Two such objects $\left\{P_{X}, c_{\alpha}\right\}$ and $\left\{P_{X}^{\prime}, c_{\alpha}^{\prime}\right\}$ are said to be equivalent if there is a bundle isomorphism over the $F_{\alpha}$ such that the vector bundle morphisms agree over the complement of some $Q_{X}$ containing both $P_{X}$ and $P_{X}^{\prime}$.

REMARK. If $f_{\alpha}$ is the induced map from $E_{\alpha}$ to $F_{\alpha}$, then by obstruction theory there is a unique factorization of $f_{\alpha} \mid E_{\alpha}-P_{X}$ through $F_{\alpha}-R_{Y}$ up to homotopy, at least if the codimension of $P_{X} \cap E_{\alpha}$ is at least three. Since in most cases the dimension of $P_{X}$ will be at most 2 and the dimension of $E_{\alpha}$ will be at least 5, this factorization exists in all cases of interest to us in this paper. We shall denote this factorization of $f_{\alpha} \mid E_{\alpha}$ $P_{X}$ by $f_{\alpha}^{\prime}$. 
Suppose now that $(F, B)$ gives a cobordism from $\left(f_{0}, b_{0}\right)$ to $\left(f_{1}, b_{1}\right)$, and let $\left(P_{l}, c_{i}\right)$ denote unstable data with deficiency $k$ for $\left(f_{i}, b_{i}\right)$, where $i=0,1$. The appropriate notion of cobordism for $\left(P_{i}, c_{i}\right)$ is a pair $(Q, C)$, where $Q$ is a $k$-saturating geometric variety of dimension $k+1$ and $C$ is unstable data for $(F, B)$, such that the restrictions to the ends of the cobordism are unstable data of deficiency $k$ and are equivalent to the $\left(P_{i}, C_{i}\right)$. (Technical note: If we would insist upon $(k+1)$-saturation for $Q$, the product of data with the unit interval would not necessarily be a cobordism.)

We define a $\mathbf{Z}_{2}$-normal map with $k$-dimensional deficiency to be a triple $(f, b, d)$, where $f$ is a degree one map, $b$ is stable bundle data for $f$, and $d$ is an equivalence class of unstable data with $k$-dimensional deficiency. Cobordisms of such objects are defined analogously. If $k<k^{\prime}$ then there is an obvious forgetful morphism from normal maps with $k$-dimensional deficiency to those with $k^{\prime}$-dimensional deficiency, and of course there are consistent forgetful morphisms from ordinary normal maps to normal maps with deficiencies. The next result says that equivariant surgery theory goes through for normal maps with deficiencies provided the dimensions of the deficiencies are not too large.

Proposition 4.4. The conclusions of Theorems $\mathrm{A}_{0}, \mathrm{~A}_{2}, \mathrm{~B}, \mathrm{C}$, and $\mathrm{C}_{8}$ all remain valid if we replace normal maps by normal maps with $k$-dimensional deficiencies, provided $k$ is less than half the dimension of each $F_{\alpha}$ for which $f_{\alpha}$ is not known to be an equivalence.

Proof. (Sketch) If we do surgery on $f_{\alpha}$ through the middle dimension, by general position we can always choose the embedded spheres to miss the geometric variety on which the unstable data are not defined. Thus for each relevant component $F_{\alpha}$ the surgery cobordism over $F_{\alpha}$ is a product over some neighborhood of $P \cap E_{\alpha}$. Using this and the unstable bundle data on the complement of $P$ one can establish a relative version of the Normal Cobordism Extension Theorem 2.2. This provides all one needs to generalize the proof of (1.2), which deals with the obstructions to doing surgery on the fixed point set; one can proceed similarly to study surgery on the fixed point set for equivariant normal maps of type $\mathbf{h}$ or $\mathbf{s}$. It remains to consider possible differences involving equivariant surgery on the complement of the fixed point set. But the entire discussion of this topic in $\$ 3$ does not use the unstable data at all, and therefore the treatment of surgery on the free part goes through unchanged.

Proposition 4.4 implies that equivariant surgery obstructions only depend upon the restriction of unstable data to the complement of a 
1-saturating geometric variety. If for some reason we know that one always has unique unstable data with 1-dimensional deficiency for some class of equivariant surgery problems, then it will follow that unstable data is not needed to define equivariant obstructions for all problems within this class. The next result proves a uniqueness theorem of this type provided either the Gap Hypothesis or the Borderline Gap Hypothesis holds.

THEOREM 4.5. Let $(f, b)$ be a degree 1 map and associated stable bundle data b, and assume either the Gap Hypothesis or the Borderline Gap Hypothesis. Then up to homotopy and equivalence there is a unique choice of unstable bundle data with 1-dimensional deficiency.

Proof. (Sketch) We begin with some general considerations. If $P$ is a $k$-saturating geometric variety for $E_{\alpha}$ and

$$
E_{\alpha}^{\prime}=E_{\alpha}-P,
$$

then by duality and the $k$-connectivity of the inclusion $E_{\alpha} \cap P \subseteq E_{\alpha}$ we know that the cohomology groups of $E_{\alpha}^{\prime}$ vanish in dimensions greater than $d(\alpha)-k-1$, where $d(\alpha)$ is the dimension of $E_{\alpha}$. This is true for twisted as well as untwisted coefficients. A similar conclusion holds with $R$ replacing $P,(k-1)$ replacing $k$, and $F_{\alpha}$ replacing $E_{\alpha}$. Denote the complement of $R$ in $F_{\alpha}$ by $F_{\alpha}^{\prime}$.

Let $f_{\alpha}^{\prime}$ be the factorization of $f_{\alpha} \mid E_{\alpha}$ through $F_{\alpha}^{\prime}$ discussed previously, and let $n$ be the dimension of $X$ and $Y$. By the remarks following Proposition 4.1 it suffices to show that the obstruction groups

$$
H^{i}\left(\operatorname{Cyl}\left(f_{\alpha}^{\prime}\right), E_{\alpha}^{\prime} ; \pi_{i-u}\left(O / O_{n-d(\alpha)}\right)\right)
$$

are zero for all values of $i$ and $u=0,1$. Since $d(\alpha) \leq(n / 2)$, the coefficients vanish unless $i=d(\alpha)+0$ or 1 . But for these choices of $i$ we know that the $(i-1)$-dimensional cohomology groups of $E_{\alpha}^{\prime}$ and the $i$-dimensional cohomology groups of $F_{\alpha}^{\prime}$ vanish by the discussion in the preceding paragraph, and therefore the relative cohomology groups vanish by exactness. One has an exact sequence even if the coefficients happen to be twisted because the twistings are given by the homomorphisms of $\pi_{1}\left(E_{\alpha}\right.$ or $\left.F_{\alpha}\right)$ into $\mathbf{Z}_{2}=\operatorname{Aut}(\mathbf{Z})$, and $f_{\alpha}$ is compatible with these maps.

\section{Final remarks}

One can also consider equivariant surgery problems for piecewise linear and topological involutions satisfying local linearity; in other words, each orbit has an invariant neighborhood that is equivariantly (piecewise 
linearly or topologically) equivalent to the total space of a $G$-vector bundle over that orbit. In particular, equivariant surgery on such $G$-manifolds is studied in papers of Dovermann and Rothenberg [14] in the topological category and Madsen and Rothenberg $[22,23]$ in the piecewise linear category when the Gap Hypothesis holds. As one would expect, these theories have many of the same formal properties as $G$-surgery theory for smooth $G$-manifolds. However, the results of Madsen and Rothenberg imply that for $G=\mathbf{Z}_{2}$ the analog of Theorem 4.5 fails systematically in both the piecewise linear and topological categories (compare [ 23], Section 4, especially Corollary 4.4). Specifically, if $V$ and $W$ are representations of the group $G=\mathbf{Z}_{2}$ and $\mathrm{CAT}_{G}$ denotes the appropriate group of CAT-equivariant automorphisms of the associated unit sphere, then there are no dimension conditions on $V$ that yield good connectivity properties for the join map from $\operatorname{CAT}_{G}(V)$ to $\operatorname{CAT}_{G}(V \oplus W)$. Thus in the nonsmooth categories one cannot expect to retrieve unstable data for PL or topological $\mathbf{Z}_{2}$-surgery from the stable data under an assumption such as the Gap Hypothesis.

Incidentally, the failure of PL and topological bundle stability is basically unique to the prime 2 . If $p$ is an odd prime and CAT refers to either the PL or topological category, then $\mathbf{Z}_{p}$-CAT bundle theory has extraordinarily good stability properties resembling those of the classical linear groups and the nonequivariant CAT bundle theories. These results are a central feature in the machinery of Madsen and Rothenberg; specifically, the case $G=\mathbf{Z}_{p}$ is treated in [22b], $\S 3$, for the piecewise linear category and $[22 \mathrm{c}], \S 7$, for the topological category.

In a very precise sense this contrast between $p=2$ and $p$ odd can be seen by comparing the desuspension results for free PL and topological $\mathbf{Z}_{p}$-actions on spheres in the cases $p=2$ and $p$ odd (for background information see [30], Chapter 14A, especially the paragraph beginning on page 184 and continuing to page 185). If $p$ is odd, then free $\mathbf{Z}_{p}$-actions usually have unique desuspensions (compare [30], Corollary, page 219), but if $p=2$ then this need not be true (compare [30], Theorem 14D.4, page 200$)$. The results of $[22 b-c]$ on stability may be viewed as multiparameter generalizations of the earlier results on desuspensions in [30].

\section{REFERENCES}

[1] G. A. Anderson, Review of [11], Math. Reviews 83b (1983) \#57019, 675-676.

[2] J. C. Becker, Cohomology and the classification of liftings, Trans. Amer. Math. Soc., 133 (1968), 447-475.

[3] G. E. Bredon, Equivariant Cohomology Theories, Lecture Notes in Mathematics Vol. 34. Springer, New York, 1967. 
[4] W. Browder, Embedding smooth manifolds, Proc. Internat. Congr. Math. (Moscow, 1966), 712-719. Izdat. “Mir”, Moscow, 1968.

[5] Surgery and the Theory of Differentiable Transformation Groups, Proceedings of the Conference on Transformation Groups (New Orleans, 1967), 1-46. Springer, New York, 1968.

[6] W. Browder and F. Quinn, A surgery theory for G-manifolds and stratified sets, Manifolds - Tokyo, 1973 (Conf. Proc., Univ. of Tokyo, 1973), 27-36. University of Tokyo Press, Tokyo, 1975.

[7] S. Cappell and J. Shaneson, The codimension two placement problem and homology equivalent manifolds, Ann. of Math., 99 (1974), 277-348.

[8] H. Cartan, Géneralités sur les espaces fibrés II, Séminaire H. Cartan de l'Éc. Norm. Sup. 1949/1950 (Espaces fibrés et homotopie), Exposé 7, 5 pp. Secrétariat mathématique, 11 rue $\mathrm{P}$. Curie, Paris, 1956. Reprinted in Séminaire Cartan, Tome 1 (1948-1951), Benjamin, New York, 1967.

[9] F. Connolly and V. Vijums, G-normal maps and equivariant homotopy theory, preprint, University of Notre Dame, 1979.

[10] A. Douady and L. Hérault, Arrondisement des variétés avec coins (Appendix to a paper by A. Borel and J.-P. Serre), Comment. Math. Helv., 48 (1973), 484-489.

[11] K. H. Dovermann, $\mathbf{Z}_{2}$-surgery theory, Michigan Math. J., 28 (1981), 267-287.

[12] K. H. Dovermann, M. Masuda, and R. Schultz, Conjugation involutions on homotopy complex projective spaces, Japanese J. Math., to appear.

[13] K. H. Dovermann and T. Petrie, G-Surgery II, Memoirs Amer. Math. Soc., 260 (1982).

[14] K. H. Dovermann and M. Rothenberg, An Equivariant Surgery Sequence and Equivariant Homeomorphism and Diffeomorphism Classification, Topology Symposium (Siegen, 1979), Lecture Notes in Mathematics Vol. 788, 257-280. Springer, New York, 1980 (The detailed version is scheduled to appear in the Memoirs of the A.M.S.).

[15] K. H. Dovermann and R. Schultz, Equivariant Surgery Theories and their Periodicity Properties, Springer Lecture Notes in Mathematics, to appear.

[16] A. Heller, On equivariant maps with spaces with operators, Ann. of Math., 55 (1952), 223-231.

[17] S. Illman, Equivariant Whitehead torsion and actions of compact Lie groups, Group Actions on Manifolds (Conference Proceedings, University of Colorado, 1983), Contemporary Math., 36 (1985), 91-106.

[18] I. James and E. Thomas, An approach to the enumeration problem for non-stable vector bundles, J. Math. Mech., 14 (1965), 485-506.

[19] S.-i. Kakutani, An application of Dovermann's $\mathbf{Z}_{2}$ surgery to 2 -dimensional complex projective spaces with the conjugate involution, Mem. Fac. Sci. Kôchi Univ. Ser. A (Math.) 5 (1984), 27-43.

[20] Y. Kitada, Orientation reversing involutions on Brieskorn spheres, preprint, Yokohama National University, 1985.

[21] P. Löffler, Homotopielineare $\mathbf{Z}_{p}$-Operationen auf Sphären, Topology, 20 (1981), 291-312.

[22] I. Madsen and M. Rothenberg, On the classification of G-shperes I: Equivariant transversality. Matematisk Institut, Aarhus Universitet, 1985/86 Preprint Series No. 1.

[23] On the classification of G-spheres II: PL automorphism groups, Matematisk Institut, Aarhus Universitet, 1985/86 Preprint Series No. 2.

[24] On the classification of G-spheres III: Topological automorphism groups, Matematisk Institut, Aarhus Universitet, 1985/86 Preprint Series No. 14. 
[25] M. Masuda, $\mathbf{Z}_{2}$ surgery theory and involutions on homotopy complex projective spaces, to appear.

[26] T. Petrie and J. Randall, Transformation Groups on Manifolds, Dekker Series in Pure and Applied Math. Vol. 82. Marcel Dekker, New York, 1982.

[27] M. Rothenberg, Torsion invariants and finite transformation groups, Proc. Amer. Math. Soc. Sympos. Pure Math. 32 Pt. 1 (1978), 267-311.

[28] R. Schultz, Exotic spheres as stationary sets of homotopy sphere involutions, Michigan Math. J., 29 (1982), 121-122.

[29] W. A. Sutherland, The fibre homotopy enumeration of nonstable sphere bundles and fibrings over real projective space, J. London Math. Soc., (2) 1 (1969), 693-704.

[30] C. T. C. Wall, Surgery on Compact Manifolds, London Math. Soc. Monographs No. 1. Academic Press, New York, 1970.

Received July 15, 1986. The first named author was partially supported by NSF grants MCS81-00751 and MCS85-14551, and the second named author was partially supported by NSF grants MCS83-00669 and MCS86-02543.

UNIVERSITY OF HAWAII

HONOLULU, HI 96825

AND

PURDUE UNIVERSITY

WEST LAFAYETTE, IN 47907 



\section{EDITORS}

\author{
V. S. VARADARAJAN \\ (Managing Editor) \\ University of California \\ Los Angeles, CA 90024 \\ HeRbert Clemens \\ University of Utah \\ Salt Lake City, UT 84112 \\ R. FINN \\ Stanford University \\ Stanford, CA 94305
}

\author{
HERMANN FLASCHKA \\ University of Arizona \\ Tucson, AZ 85721 \\ RAMESh A. GANGOLLI \\ University of Washington \\ Seattle, WA 98195 \\ VAUGHAN F. R. JONES \\ University of California \\ Berkeley, CA 94720
}

\author{
ROBION KIRBY \\ University of California \\ Berkeley, CA 94720 \\ C. C. MOORE \\ University of California \\ Berkeley, CA 94720 \\ HAROLD STARK \\ University of California, San Diego \\ La Jolla, CA 92093
}

\section{ASSOCIATE EDITORS}
R. ARENS
E. F. BECKENBACH
B. H. NEUMANN
F. WOLF
K. YOSHIDA
(1906-1982)

\section{SUPPORTING INSTITUTIONS}

UNIVERSITY OF ARIZONA
UNIVERSITY OF BRITISH COLUMBIA
CALIFORNIA INSTITUTE OF TECHNOLOGY
UNIVERSITY OF CALIFORNIA
MONTANA STATE UNIVERSITY
UNIVERSITY OF NEVADA, RENO
NEW MEXICO STATE UNIVERSITY
OREGON STATE UNIVERSITY

\author{
UNIVERSITY OF OREGON \\ UNIVERSITY OF SOUTHERN CALIFORNIA \\ STANFORD UNIVERSITY \\ UNIVERSITY OF HAWAII \\ UNIVERSITY OF TOKYO \\ UNIVERSITY OF UTAH \\ WASHINGTON STATE UNIVERSITY \\ UNIVERSITY OF WASHINGTON
}

The Supporting Institutions listed above contribute to the cost of publication of this Journal, but they are not owners or publishers and have no responsibility for its content or policies.

Mathematical papers intended for publication in the Pacific Journal of Mathematics should be in typed form or offset-reproduced (not dittoed), double spaced with large margins. Please do not use built up fractions in the text of the manuscript. However, you may use them in the displayed equations. Underline Greek letters in red, German in green, and script in blue. The first paragraph must be capable of being used separately as a synopsis of the entire paper. In particular it should contain no bibliographic references. Please propose a heading for the odd numbered pages of less than 35 characters. Manuscripts, in triplicate, may be sent to any one of the editors. Please classify according to the scheme of Math. Reviews, Index to Vol. 39. Supply name and address of author to whom proofs should be sent. All other communications should be addressed to the managing editor, or Elaine Barth, University of California, Los Angeles, California 90024.

There are page-charges associated with articles appearing in the Pacific Journal of Mathematics. These charges are expected to be paid by the author's University, Government Agency or Company. If the author or authors do not have access to such Institutional support these charges are waived. Single authors will receive 50 free reprints; joint authors will receive a total of 100 free reprints. Additional copies may be obtained at cost in multiples of 50 .

The Pacific Journal of Mathematics is issued monthly as of January 1966. Regular subscription rate: $\$ 190.00$ a year (5 Vols., 10 issues). Special rate: $\$ 95.00$ a year to individual members of supporting institutions.

Subscriptions, orders for numbers issued in the last three calendar years, and changes of address should be sent to Pacific Journal of Mathematics, P.O. Box 969, Carmel Valley, CA 93924, U.S.A. Old back numbers obtainable from Kraus Periodicals Co., Route 100, Millwood, NY 10546.

The Pacific Journal of Mathematics at P.O. Box 969, Carmel Valley, CA 93924 (ISSN 0030-8730) publishes 5 volumes per year. Application to mail at Second-class postage rates is pending at Carmel Valley, California, and additional mailing offices. Postmaster: send address changes to Pacific Journal of Mathematics, P.O. Box 969, Carmel Valley, CA 93924.

PUBLISHED BY PACIFIC JOURNAL OF MATHEMATICS, A NON-PROFIT CORPORATION Copyright (c) 1987 by Pacific Journal of Mathematics 


\section{Pacific Journal of Mathematics}

\section{Vol. 130, No. $2 \quad$ October, 1987}

Bernhard Banaschewski, J. L. Frith and C. R. A. Gilmour, On the congruence lattice of a frame ............................ 209

Paul S. Bourdon, Density of the polynomials in Bergman spaces ........2215

Lawrence Jay Corwin, Approximation of prime elements in division

algebras over local fields and unitary representations of the multiplicative group

Stephen R. Doty and John Brendan Sullivan, On the geometry of extensions of irreducible modules for simple algebraic groups

Karl Heinz Dovermann and Reinhard Schultz, Surgery of involutions

with middle-dimensional fixed point set ....................2 275

Ian Graham, Intrinsic measures and holomorphic retracts ............ 299

John Robert Greene, Lagrange inversion over finite fields . . . . . . . . . . . 313

Kristina Dale Hansen, Restriction to $\mathrm{GL}_{2}(\mathrm{O})$ of supercuspidal

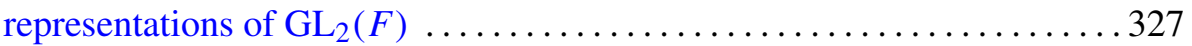

Kei Ji Izuchi, Unitary equivalence of invariant subspaces in the polydisk ....351

A. Papadopoulos and R. C. Penner, A characterization of pseudo-Anosov

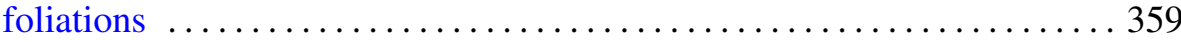

Erik A. van Doorn, The indeterminate rate problem for birth-death

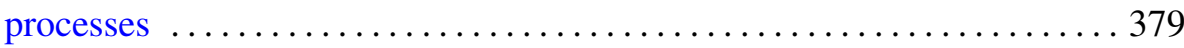

Ralph Jay De Laubenfels, Correction to: "Well-behaved derivations on

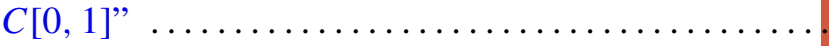

Robert P. Kaufman, Correction to: "Plane curves and removable sets" . . . . 396

Richard Scott Pierce and Charles Irvin Vinsonhaler, Correction to:

"Realizing central division algebras" 